\title{
OVINE RICKETS
}

By Dr. A. B. GRANT, Biochemist, Wallaceville Animal Research Station, Dept. of Agriculture.

Rickets is common in New Zealand lambs which are grazing on winter greenfeeds, the incidence being highest on cereal crops, lowest on brassicas, while ryegrasses appear to lie between these extremes in rachitogenic potency. The disease is more common in the milder seasons which favour good growth during late autumn and early winter. The presence of a rachitogenic substance in greenfeeds was first postulated by Fitch, and the results of subsequent work has not only confirmed his opinion but indicated that carotene, the main plant carotenoid precursor of vitamin A, is the main factor involved. The rachitogenic properties of vitamin $\mathrm{A}$ or its precursors have been described recently, and it is apparent that the higher the vitamin A uptake the greater will be the vitamin D requirement of the animal.

Rickets is primarily the result of a vitamin D deficiency but, as well as avitaminosis D, lack of adequate dietary calcium or phosphorus can adversely affect bone calcification and so also can any substance or circumstance which impairs utilization of either of these elements. Until recently it has been assumed that vitamin A directly inhibited vitamin D function, for the earlier field trials and observations indicated that supplements rich in calcium and phosphorus were ineffective in reducing the severity of rickets in lambs. This view was supported by the results of earlier laboratory trials with rats which suggested a quantitative relationship between intake of vitamin $\mathrm{A}$ and its resulting inhibitory effect on the bone calcifying properties of vitamin D. More recent findings however (unpublished) do not bear out this hypothesis but point rather to vitamin A affecting some other factor involved in bone formation, and it is hoped that laboratory investigations being carried out at the present time will clear up this point.

From determination of the carotene content of a number of fodder plants it seems probable that most good quality greenfeeds have a similar carotene content and sheep grazing upon them will have a daily carotene intake approximately equivalent to $1-2$ million I.U. 
vitamin A. Daily 'vitamin A doses of this magnitude, quite apart from their pronounced rachitogenic effect, were found to be sufficient to produce in lambs symptoms of hypervitaminosis A involving depressed growth rate, unthrifty apearance, osteoporosis and lowering of blood phosphorus levels. Since this picture closely resembled that associated with the common disease known as "lamb or hogget ill-thrift" it became a matter of importance to determine the amount of carotene converted to vitamin $\mathrm{A}$ in the case of animals grazing good green pastures.

Approximate estimates made from the results of same preliminary trials suggest that only 1 per cent to 5 per cent of the ingested carotene is utilised by the sheep so that vitamin A uptake is not of that order which might produce symptoms of vitamin A poisoning. This relatively low rate of conversion of pasture carotene to vitamin A however could still result in a sufficiently high daily uptake of the vitamin to induce a marked rachitogenic effect in lambs if it can be assumed that the rachitogenic potency of vitamin $A$ parallels that which has been observed in the rat.

The reason why same fodder craps such as green cereals are much more rachitogenic than other greenfeeds may lie in a variation of carotene availability with plant species or with the stage of growth at which these craps are normally grazed, but further investigation is required before this question can be satisfactarily answered.

From the fact that the Iamb in most New Zealand latitudes cannot rely on winter solar ultraviolet irradiation to provide adequate body synthesis of vita-. min D, and since the green pasture leaf is practically devoid of this vitamin, it is not unlikely that a large number of our sheep are law in vitamin D status at this time of the year. In the majority of cases the balance is probably maintained by the fact that, when pasture becomes scarce, a retardation in growth rate will reduce vitamin $\mathrm{D}$ requirement, or when a winter supplement of hay is made available vitamin D intake is thereby increased.. When attempts are made to maintain maximum growth rate throughout the winter months, however, by grazing greenfeed or autumn saved pasture, which provides no vitamin $\mathrm{D}$ and an appreciable uptake of Vitamin A, it is not. surprising that the result is frequently an outbreak of clinical rickets.

Rickets in a mildly affected flock is almost impassible to diagnose in the field but moderate to severe eases can be readily detected by a characteristic stiff- 
ness in gait, or lameness of the affected animals. The disease is accompanied by what has been termed "illthrift." This is probably better described as a slackening off in normal growth rate. The results of a number of trials carried out in recent years indicate that the growth rate of lambs affected with mild to moderate rickets will be reduced by only about $25 \%$, and even in moderate to severe outbreaks the average growth rate of the flock is seldom more than halved. An actual cessation of growth or decline in body weight resulting from rickets is probably confined to a few cases in which 'grazing has been restricted by severe lameness.

The disease can be prevented or rapidly cured by administering a single $\frac{1}{2} \mathrm{oz}$. oil drench containing $\mathbf{1 , 0 0 0 , 0 0 0}$ I.U. calciferol. This dose normally ensures complete winter protection from rickets for New Zealand lambs. From what has been said it would seem sound practice to administer ca.lciferol to all lambs which are, to be allowed the winter grazing of young cereal crops or particularly good quality pastures.

Under no other conditions can the use of calciferol be recommended as a preventive measure for if lambs do not have access to winter greenfeed development of rickets to a serious degree is unlikely. If a moderate or severe outbreak does occur under such conditions it can be readily detected by the presence of stiff or lame animals in the flock. Drenching with calciferol at this stage would be soon enough to avoid appreciable economic- loss. The development' of very mild rickets in which rachitic lameness is not evident may. be common in lambs which have had no winter greenfeed grazing but..even if these cases could be readily diagnosed, the value of calciferol in stimulating growth to the extent of only a few pounds is probably not worth the cost and labour involved, particularly as the small reduction in growth rate of affected animals tends to be made up during the following spring and summer.

The retardation of growth rate associated with rickets is in no way comparable with the growth picture presented by another common disease known as "lamb ill-thrift". The latter usually results in a complete cessation of growth or a rapid decline in body weight and a marked unthrifty appearance of affected animals. Lameness is not one of the characteristic features. Although osteoporosis and low blood phosphorus can accompany this condition it appears to be connected in no way with a failure in vitamin $D$ function and these cases do not respond favourably to calciferol. On the contrary calciferol given to these animals at 
the rate of $1,000,000$ units per dose may do more harm than good for recent trials have indicated that this massive vitamin $\mathrm{D}$ dose has some toxic effect which some unthrifty lambs may be unable to tolerate. Results of these trials are depicted graphically in Fig. 1 which depicts the results of three trials in which avarage weight gain of calciferol drenched lambs is directly compared with that of control group animals. Due to the frequent rains during the period when these trials were being conducted varying fleece water content was the main cause of the gross irregularities observed in the weight gains and in order to clarify the picture these have been ironed out as illustrated in Fig. 2 which shows the depression in average body weight gain of calciferol treated animals compared with that of the control group, which in every case is represented
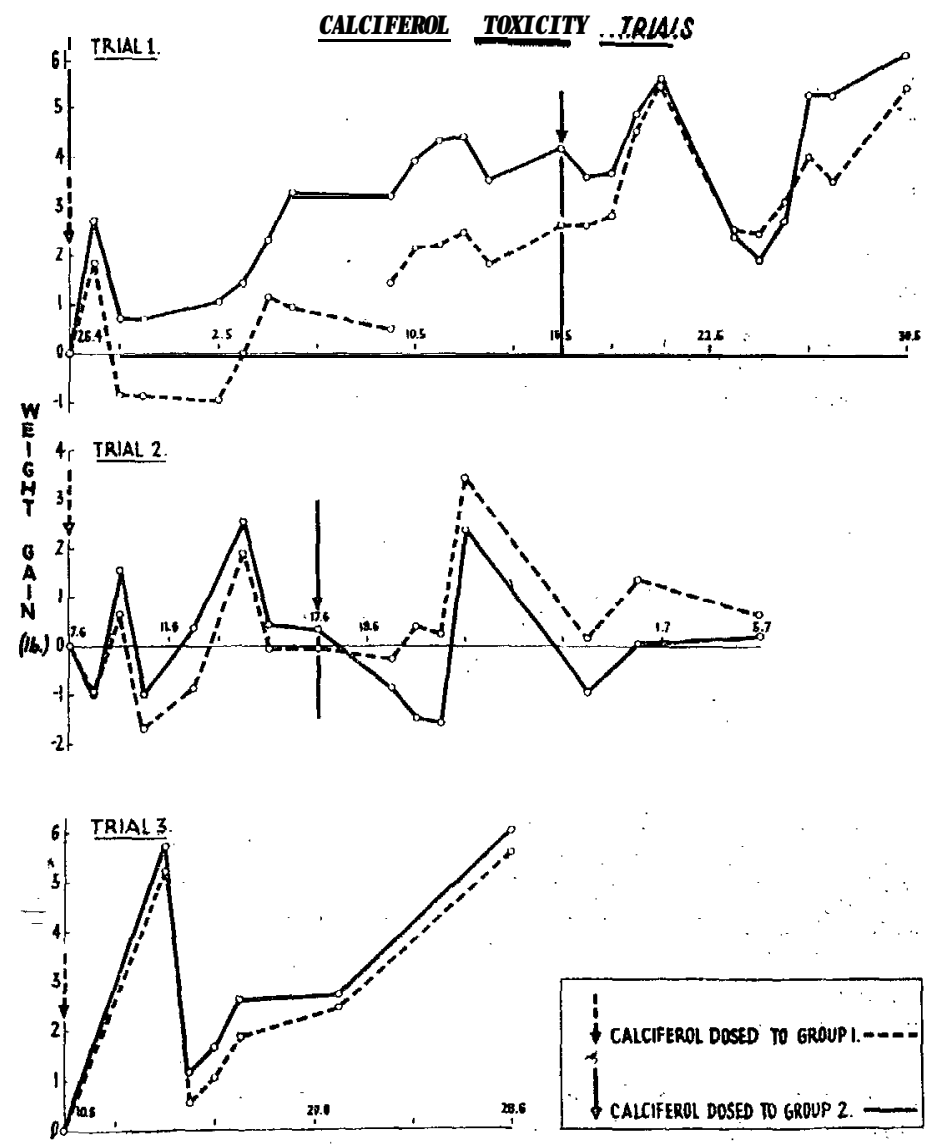

Figure 1 . 


\section{CALCIFEROL TOXICITY TRIALS.}

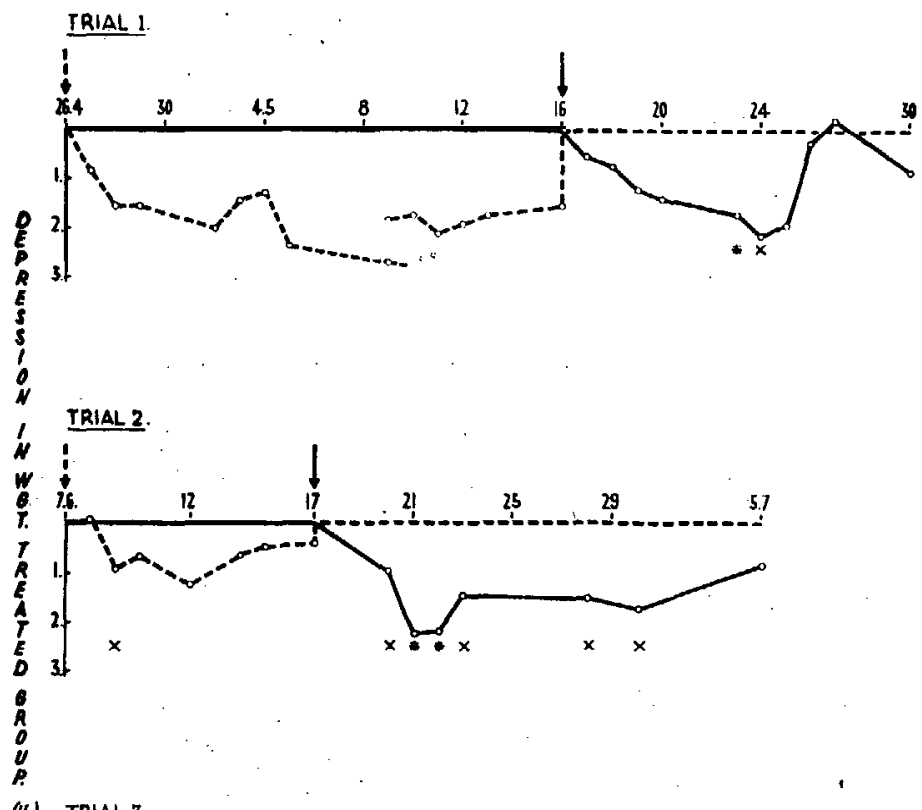

ab) TRIAL 3

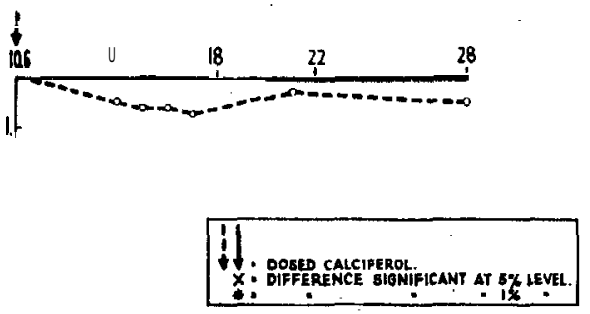

Figure 2.

by the straight horizontal line. The animals employed in trials 1 and 2 had suffered appreciably from "lamb ill-thrift" but when these trials began the most serious phase of the disease involving rapid losses in body weight and high mortality rate in the flocks had passed. Resumption in body weight gains had commenced in most of the animals of trial 1 but there was still a marked individual variation in body weight changes in the flock used for trial 2. In the latter case 11 animals had lost some weight while the remaining 15 had either maintained or increased body weight over the period of 8 days immediately prior to commencement of the trial. 
Each flock was divided at random into two groups. The animals of one group were each given 1,000,000 I.U. calciferol in a $\frac{1}{2}$ oz. oil drench while the other group received no supplement. When it was considered that the initial toxic effect of the calciferol dose had passed the control group animals were given the same calciferol treatment and the group which had previously been dosed then acted as controls. This crossover technique greatly increased the probability that calciferol treatment was in fact the cause of the observed differences between the groups.

\section{In Trial 1:-}

During the first stage on $9 / 5,4$ of the 7 animals of the treated group had lost weight while only 1 of the 7 control animals had suffered loss.

In the second stage on $23^{\circ} / 5,6$ of the 7 treated animals had lost weight as compared with 2 of the 6 controls (one of the animals had died during the first stage).

In Trial 2:-

In the first stage on $12 / 6,12$ out of 14 calciferol treated lambs had lost weight while only 3 of the 12 control animals had lost.

And in the second stage on 22/6, all 12 animals of the treated calciferol group had lost weight while only 4 of the 14 controls had similarly suffered.

The occasions on which the difference between groups was statistically significant are denoted on the graphs by asterisks.

For trial 3 eighteen thrifty lambs of an average weight of $881 \mathrm{~b}$. were employed. These were growing rapidly and had suffered no appreciable setback during the autumn. Since previous laboratory trials had indicated that, whereas a massive calciferol dose appreciably affected growth rate in unhealthy rats a similar dose was well tolerated by the thrifty animal, the nine calciferol drenched sheep were each given 2,000,000 I.U. in a single dose. These lambs apparently tolerated the dose quite well and the maximum retardation in average growth rate to the extent of only $0.72 \mathrm{lb}$. was observed on 17/6. At no time was the difference between groups statistically significant.

It seems obvious that, whereas a massive calciferol dose will have no appreciable adverse effect on the healthy lamb, the toxic effect on the unthrifty animal can be marked. The common practice of drenching calciferol to animals suffering from "lamb ill-thrift". 
can therefore be condemned particularly in the case of flocks suffering a high mortality rate where the effect of such treatment is likely only to increase the loeses. Unless rachitic lameness is a marked feature in a flock of unthrifty lambs the cause of the disorder is almost certainly not a lack of vitamin $\mathrm{D}$ and no benefit from calciferol can be expected.

Pasture carotene is not utilised to an excessive degree and its value as the main source of vitamin A for farm stock probably far outweighs its ill-effects as a rachitogenic factor. In view of these considerations, and since it apears likely that the density of green leaf pigments prevents vitamin D synthesis by shielding the precursor from ultraviolet light, it is difficult to see how the plant breeder could provide a simple solution. It seems that the problem of rickets must remain largely in the hands of the veterinarian, and his advice in the matter of calciferol administration can do much to prevent the disappointing and even harmful effect of its misuse and thus ensure that this valuable product does not fall into disrepute in the opinion of the sheep breeder.

\section{DISCUSSION}

Q. Please clarify the difference between rickets and lamb “illthrift", aarticularly in resnect of time of occurrence?

A. Lamb "ill-thrift". usually occurs following an autumn flush of feed and is common in May and June. Recovery is spontaneous. Calciferol has been used by some farmers in an endeavour to cure the "ill-thrift". The sheen recover in spite of rather than because of the calciferol.

Q. When do rickets become apparent at Wallaceville ? In Canterbury they are apparent in July.

'4. Cases with symptoms of marked lameness have been seen as early as May when the lambs are on green feed.

Q. A concentration of vitamin A is being sold for treating lambs at tailing and at weaning. If vitamin $\mathrm{A}$ is abundant in the green leaves of plants is such a concentrate of any value?

A. Not one bit.

Q. Is it possible that dosing ewes in June will have a beneficial effect on the lamb before or after birth?

A. It would have no effect on the lamb after 'birth.

Q. Please define osteoporosis ?

A. This is the decalcification of the bones. The ribs become very fragile and are not unlike cardboard in the advanced stages. In rickets the lamb is lame and the bones are enlarged.

Q. What is the effect of a mild attack of rickets?

A. The lambs recover by August and there is no after effect. 